\title{
Interfacial spin-orbit splitting and current-driven spin torque in anisotropic tunnel junctions
}

\author{
A. Manchon \\ Division of Physical Science and Engineering, Materials Science and Engineering, KAUST, Thuwal 23955-6900, Saudi Arabia
}

(Received 21 March 2011; published 17 May 2011)

\begin{abstract}
Spin transport in magnetic tunnel junctions comprising a single magnetic layer in the presence of interfacial spin-orbit interaction (SOI) is investigated theoretically. Due to the presence of interfacial SOI, a current-driven spin torque can be generated at the second order in SOI, even in the absence of an external spin polarizer. This torque possesses two components, one in plane and one perpendicular to the plane of rotation, that can induce either current-driven magnetization switching from an in-plane to out-of-plane configuration or magnetization precessions, similar to spin transfer torque in spin valves. Consequently, it appears that it is possible to control the magnetization steady state and dynamics by either varying the bias voltage or electrically modifying the SOI at the interface.
\end{abstract}

DOI: 10.1103/PhysRevB.83.172403

PACS number(s): 75.47.-m, 72.25.-b, 73.40.Rw, 73.43.Qt

Since the theoretical prediction and experimental observation of current-driven magnetization control, ${ }^{1,2}$ spintronics is considered to be a promising candidate for low-energyconsumption devices. ${ }^{3}$ Up until now, spin transfer torque (STT) has essentially been observed in inhomogeneous magnetic structures such as spin valves, magnetic tunnel junctions (MTJs), and magnetic domain walls. ${ }^{4}$ Moreover, recent experimental observations on single ferromagnet-based structures have opened promising opportunities for new device concepts. For example, tunneling anisotropic magnetoresistance (TAMR) has been observed in MTJs comprising a single ferromagnet ${ }^{5-8}$ (referred to as semi-MTJs, SMTJs). Reference 9 proposed to exploit the spin-orbit interaction (SOI) present in a single ferromagnetic layer to electrically control the magnetization direction. ${ }^{10,11}$ Alternatively, the voltage-controlled manipulation of magnetic anisotropy of thin magnetic layers through thick insulators has been achieved. ${ }^{12}$ Efficient electrical control of the magnetization direction of a single ferromagnetic layer combined with a sizable TAMR effect would offer powerful perspectives for spin-based memory devices.

The key ingredient of TAMR,${ }^{5-8}$ SOI-induced torque (SOI-ST), ${ }^{9}$ and magnetic anisotropy ${ }^{12}$ is the SOI arising either from bulk inversion asymmetry ${ }^{11}$ or from structure inversion asymmetry. ${ }^{10,12}$ In the latter case, the presence of a large potential gradient $\nabla V$ at the interface between, say, an insulator and a metal generates a local electric field perpendicular to the interface $\nabla V=-E \mathbf{z}$, inducing a SOI of the form ${ }^{13}$

$$
\hat{H}_{R}(\mathbf{k})=\mathbf{B}_{R}(\mathbf{k}) \cdot \hat{\boldsymbol{\sigma}}=\alpha_{R}(\mathbf{k} \times \mathbf{z}) \cdot \hat{\boldsymbol{\sigma}}
$$

The precise form and magnitude of $\alpha_{R}$ terms has been widely studied in semiconductor two-dimensional electron gases ${ }^{13,14}$ and has only recently been studied at metallic interfaces and can be as large as $1-5 \mathrm{eV} \AA^{2}$. 6,15

In this Brief Report, we suggest that the presence of such an interfacial SOI at the interface between a ferromagnet and an insulator in a SMTJ is responsible for a nonequilibrium spin torque. We demonstrate that the magnetization of the ferromagnetic layer of such a junction can be controlled or excited by an external bias voltage applied across the junction. In the nonequilibrium regime, the interfacial SOI generates a bias-voltage-driven spin torque on the ferromagnetic layer, inducing either magnetization switching or self-sustained magnetic precessions. This effect belongs to the family of SOI-induced spin torques, ${ }^{9,16}$ and the correspondences with the conventional STT observed in spin valves ${ }^{1,2}$ will be discussed at the end of this Brief Report.

In regular MTJs, the transport is essentially controlled by the interfacial densities of states. ${ }^{17}$ Therefore, the tunneling magnetoresistance (TMR) and STT are proportional to the interfacial polarization of the electrodes $P_{i}:$ TMR $\propto P_{L} P_{R}$, $\tau_{\|} \propto P_{L} \sin \theta$, and $\tau_{\perp} \propto P_{L} P_{R} \sin \theta .{ }^{17}$ In contrast, in the case of SMTJs, $P_{L}=0$, and both STT and TMR should vanish. However, in the presence of interfacial SOI, it has been found that the resistance of the junction depends on the orientation of the magnetization against the interface. ${ }^{5}$

This TAMR effect has been observed using semiconductors, ${ }^{5}$ metals, ${ }^{7}$ and hybrid structures. ${ }^{6,8}$ Theoretical investigations ${ }^{6,18}$ have shown that TAMR is well described with Rashba and Dresselhaus effective $\mathrm{SOI}^{13,19}$ (the latter being restricted to noncentrosymmetric crystals). Following Eq. (1), Rashba SOI induces an angular

(a)
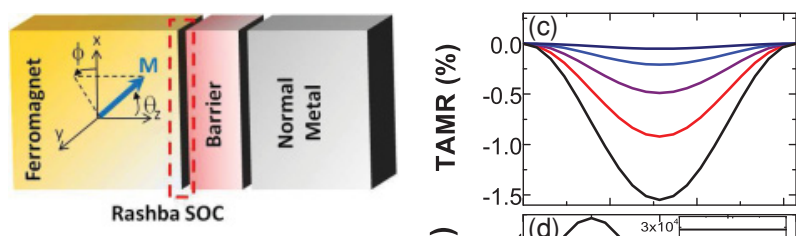

(b)
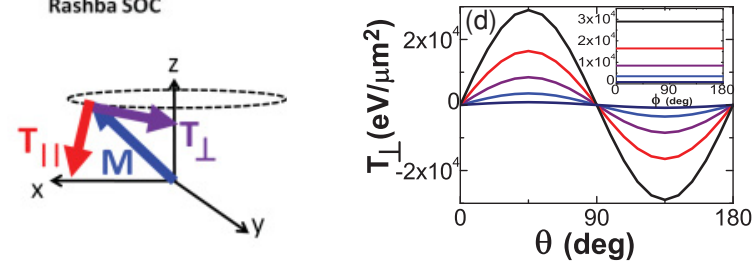

FIG. 1. (Color online) (a) Schematics of a semimagnetic tunnel junction. (b) Influence of the spin torque on the magnetization. Angular dependence of (c) TAMR and (d) perpendicular torque $T_{\perp}$ at zero bias for a rotation in the (010) planes and $\alpha_{R} \in[1,5] \mathrm{eV} \AA^{2}$. The parameters are adapted for an Fe-MgO interface: $U_{0}=1 \mathrm{eV}$, $d=0.6 \mathrm{~nm}, k_{F}^{\uparrow}=1.09 \mathrm{~nm}^{-1}, k_{F}^{\downarrow}=0.4 \mathrm{~nm}^{-1}$. Inset in (d) shows $\phi$ dependence of the perpendicular torque. 
momentum transfer from the spin momentum to the orbital degree of freedom. Since the interfacial SOI is linear in $\mathbf{k}$, this effect vanishes at the first order in SOI after averaging over the Fermi sphere $\left[\left\langle\hat{H}_{R}(\mathbf{k})\right\rangle=0\right]$. But in a ferromagnet, due to the presence of the local magnetic exchange, a net transfer of angular momentum occurs at the second order $\left[\left\langle\hat{H}_{R}(\mathbf{k})^{2}\right\rangle \neq 0\right]$. As a consequence, magnetic anisotropy and TAMR appear, proportional to the second order in Rashba SOI, $\propto \alpha_{R}^{2} .{ }^{18,19}$ Interestingly, since the angular momentum transfer involves only in-plane components of the spin and orbital momentum (i.e., $\sigma_{x, y}$ and $k_{x, y}$ ), the itinerant spin density produced by this transfer lies in the $(x, y)$ plane only.

In the present work, we consider the $F / I / N$ trilayer depicted in Fig. 1, where $F$ is a ferromagnetic layer (Co, Fe, $\mathrm{Ni}$, and compounds), $I$ is an insulator ( $\mathrm{MgO}, \mathrm{AlOx}, \mathrm{GaAs})$, and $N$ is a normal metal $(\mathrm{Cu}, \mathrm{Ag}, \mathrm{Au}, \mathrm{Pt}$, etc.). In order to capture the most relevant features of the mechanism described here, we choose a minimal model only considering the most pertinent material parameters. Matos-Abiague et al. ${ }^{18}$ showed that, in the case of centrosymmetric barriers (such as AlOx, $\mathrm{MgO}$ ), the TAMR is mostly due to the Rashba SOI at the interface between the ferromagnet and the tunnel barrier. The free-electron Hamiltonian of the junction is then

$$
\hat{H}=-\frac{\hbar^{2}}{2} \nabla \frac{1}{m(z)} \nabla+U(z)+\hat{H}_{R} \delta\left(z-z_{L}\right) .
$$

$\hat{H}_{R}$ is given in Eq. (1), and $m(z)$ is the effective mass of the electron, equal to $m_{0}$ in the electrodes and $m_{\text {eff }} m_{0}$ in the barrier. $U(z)$ is the potential of the junction, given by

$$
\begin{gathered}
U_{z<0}=J \hat{\boldsymbol{\sigma}} \cdot \mathbf{M}+\frac{e V_{b}}{2}, U_{z>d}=-\frac{e V_{b}}{2}, \\
U_{0<z<d}=U_{0}+\left(\frac{1}{2}-\frac{z}{d}\right) e V_{b},
\end{gathered}
$$

where $U_{0}$ and $d$ are the barrier height and thickness, $V_{b}$ is the bias voltage, $J$ is the $s$ - $d$ exchange coupling, $\hat{\sigma}$ is the vector of Pauli spin matrices, and $\mathbf{M}=(\sin \theta \cos \phi, \sin \theta \sin \phi, \cos \theta)$ is the magnetization direction of the ferromagnetic electrode (see Fig. 1).

The mechanism giving rise to itinerant spin density can be understood by looking at the spin-density continuity equation $\frac{d \mathbf{m}}{d t}=\frac{1}{i \hbar}\langle[\hat{\boldsymbol{\sigma}}, \hat{H}]\rangle$, which reads

$$
\frac{d \mathbf{m}}{d t}=\frac{i \hbar}{m} \nabla \cdot\langle\hat{\boldsymbol{\sigma}} \otimes \nabla\rangle-\frac{2 J}{\hbar} \mathbf{m} \times \mathbf{M}+\frac{1}{i \hbar}\left\langle\left[\hat{\boldsymbol{\sigma}}, \hat{H}_{R}\right]\right\rangle .
$$

The first term is the regular spin-current divergence in the absence of SOI, the second term is the torque exerted by the itinerant spin density $\mathbf{m}$ on the local magnetization $\mathbf{M}$, and the last term is the torque between the itinerant spin and orbital angular momentum. For instance, in MTJs the last term is generally zero, and the spin torque is directly associated with the spatial variation of the spin current $\propto\langle\hat{\boldsymbol{\sigma}} \otimes \nabla\rangle .{ }^{17}$ In the present case, Rashba SOI acts like a source for spin density, and therefore, the spin torque is no longer simply related to the spin current. Consequently, the proper way to evaluate the spin torque is to calculate directly the local itinerant spin density $\mathbf{m}(z)$. We assume a semi-infinite magnetic layer, as is usually done in magnetic tunnel junctions: ${ }^{17}$ The total spin torque exerted by the transverse spin density $\mathbf{m}$ on the magnetization
$\mathbf{M}$ is defined as $\mathbf{T}=2 J / \hbar \int_{V} \mathbf{m} \times \mathbf{M} d V$, where $V$ is the volume of the magnetic layer.

The charge and spin currents are then evaluated using the conventional definitions

$$
\begin{gathered}
J_{e}=\frac{e}{\hbar} \Im\left[\sum_{s, i} \int d E d^{2} \mathbf{k}_{\|} \Psi_{i}^{s *}\left(E, \mathbf{k}_{\|}\right) \partial_{z} \Psi_{i}^{s}\left(E, \mathbf{k}_{\|}\right) f_{i}\right], \\
\mathbf{m}=\sum_{s, i} \int d E d^{2} \mathbf{k}_{\|} \Psi_{i}^{s *}\left(E, \mathbf{k}_{\|}\right) \hat{\boldsymbol{\sigma}} \Psi_{i}^{s}\left(E, \mathbf{k}_{\|}\right) f_{i},
\end{gathered}
$$

with $\Psi_{i}^{s}\left(E, \mathbf{k}_{\|}\right)$being the Hartree-Fock two-component wave function for an electron of energy $E$ and in-plane wave vector $\mathbf{k}_{\|}$, issued from the $i$ th reservoir with a Fermi-Dirac distribution $f_{i}$. Assuming convenient material parameters (barrier characteristics and effective mass), the present minimal model satisfyingly reproduces the TAMR obtained by Matos-Abiague et al. ${ }^{6,18}$ in the case of $\mathrm{Fe} / \mathrm{GaAs} / \mathrm{Au}$ and the one obtained by Park et al. ${ }^{7}$ in $(\mathrm{Pt} / \mathrm{Co})_{n} / \mathrm{AlOx} / \mathrm{Pt}$, with a reasonable degree of accuracy. The angular dependence of the zero-bias conductance for rotation of the magnetization in the (010) plane for $\alpha_{R}=1-5 \mathrm{eV} \AA^{2}$ is displayed in Fig. 1(c) and shows the expected $(\cos 2 \theta-1)$ dependence. ${ }^{6,18}$ This angular dependence can be understood by noticing that the junction is physically equivalent upon the transformation $\theta \rightarrow-\theta \rightarrow \theta+\pi .^{18}$

Since the torque is, by definition, perpendicular to the magnetization, it reads

$$
\mathbf{T}=T_{\|} \mathbf{M} \times(\mathbf{z} \times \mathbf{M})+T_{\perp} \mathbf{z} \times \mathbf{M} .
$$

The spin torque possesses two components, which can be referred to as in-plane torque $T_{\|}$and perpendicular torque $T_{\perp}$. At zero bias, only the perpendicular torque $T_{\perp}$ is nonzero, and it displays an angular dependence on the form $\sin 2 \theta$ [Fig. 1(d)], which favors the perpendicular configuration. As mentioned above, the itinerant spin density due to Rashba SOI lies in the $(x, y)$ plane of the junction [Eq. (1)]. Consequently, (i) when the magnetization is oriented along $\mathbf{z}(\theta=0)$, no transfer occurs $\left(\left\langle\sigma_{x, y}\right\rangle=0\right)$, and the spin torque vanishes; (ii) when the magnetization is oriented in the $(x, y)$ plane $(\theta=\pi / 2)$, since the spin density lies itself in this plane, the spin torque is also zero. Finally, the junction is invariant under $\phi$ rotation, so the spin torque (and resistance) does not depend on $\phi$ [Fig. 1(d), inset]. This gives rise to an angular dependence on the form $\sin 2 \theta$ as well as four associated stable magnetic states: two perpendicular to the plane $(\theta=0, \pi)$ and two in the plane $(\theta= \pm \pi / 2)$.

When applying a bias voltage across the junction, spinpolarized electrons tunnel through the barrier, significantly modifying the spin imbalance in the left electrode. Therefore, both the TAMR and the magnetic anisotropy are strongly affected by the voltage. In Fig. 2 the nonequilibrium in-plane torque $T_{\|}\left(V_{b}\right)$ is represented a function of $\alpha_{R}$ [Fig. 2(a)] for different bias voltages and as a function of the voltage [Fig. 2(b)] for different $\alpha_{R}$. The spin torque displays a quadratic dependence on the Rashba parameter, as expected from the symmetry of the Rashba Hamiltonian (linear in $\mathbf{k}$ ). For larger Rashba parameters, higher orders in $\alpha_{R}$ appear (not shown). Interestingly, the amplitude of the nonequilibrium perpendicular torque $T_{\perp}\left(V_{b}\right)-T_{\perp}(0)$ is about one order of 


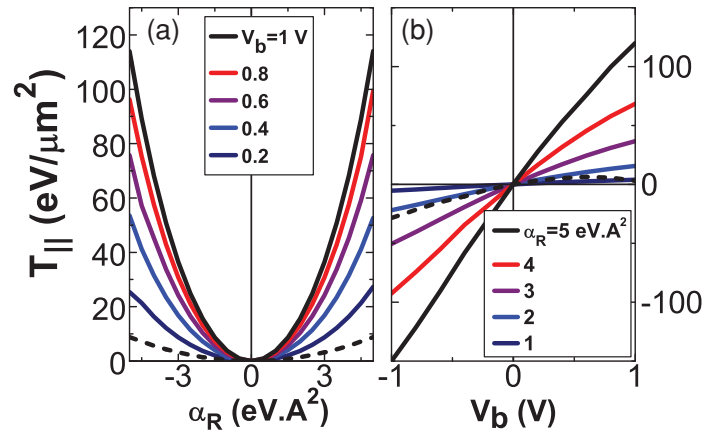

FIG. 2. (Color online) Nonequilibrium in-plane torque $T_{\|}\left(V_{b}\right)$ as a function of (a) the Rashba parameter and (b) bias voltage. The dashed line shows the nonequilibrium perpendicular torque for $V_{b}=$ $0.8 \mathrm{~V}$ in (a) and $a_{R}=4 \mathrm{eV} \AA^{2}$ in (b). The parameters are the same as in Fig. 1.

magnitude smaller than the in-plane torque [dashed lines in Figs. 2(a) and 2(b)].

The form of the torque displayed in Eq. (8) is similar to the usual STT in a MTJ whose polarizer is oriented along $\mathbf{z} .{ }^{17}$ Whereas the perpendicular torque $\propto \mathbf{z} \times \mathbf{M}$ competes with the demagnetizing field and the perpendicular anisotropy, the in-plane torque $\propto \mathbf{M} \times(\mathbf{z} \times \mathbf{M})$ competes with the damping. As a consequence, one expects the current-driven torque to produce current-induced magnetization switching from out of plane to in plane and vice versa as well as current-driven magnetization precessions.

In order to illustrate the current-driven magnetization dynamics that can be generated in such structures, we numerically solve the macrospin Landau-Lifshitz-Gilbert equation in the presence of spin torque using a fourth-order Runge-Kutta method. ${ }^{4}$ A ferromagnetic layer is considered, possessing both in-plane and out-of-plane magnetic anisotropies $Q_{\|}=$ $H_{\|}^{K} / M_{s}$ and $Q_{\perp}=H_{\perp}^{K} / M_{s}$, where $H_{\|}^{K}\left(H_{\perp}^{K}\right)$ is the in-plane (perpendicular) anisotropy field and $M_{S}$ is the saturation magnetization. Both anisotropies are needed in order to achieve stable in-plane and out-of-plane magnetization states. Since $T_{\perp}<<T_{\|}$, the nonequilibrium perpendicular torque will be disregarded. In addition, no external field is applied, and only the in-plane torque $\tau_{\|}=T_{\|} /\left(\mu_{0} M_{s}^{2} d\right)$ is considered. Finally, for the numerical simulations, we adopt parameters close to the one measured by Ikeda et al. ${ }^{20}$ (see Fig. 3).

Figure 3 displays the normalized resistance of the junction $(\propto \cos 2 \theta)$ taken at a time $t=35 \mathrm{~ns}$ for a magnetization initially perpendicular to the plane. Similar results are found when the magnetization is initially in plane (not shown). Interestingly, four zones can be distinguished. For large positive in-plane torque, $\tau_{\|} \geqslant \tau_{t h}=\eta\left(1+Q_{\|}-Q_{\perp}\right)(\eta$ is the Gilbert damping), the magnetization is stable in the perpendicular direction. When $\tau_{\|} \leqslant \tau_{t h}$, out-of-plane [Fig. 4(a)] and inplane [Fig. 4(b)] magnetization precessions appear, while for even larger negative in-plane torque the in-plane anisotropy overcomes the perpendicular anisotropy and in-plane stable states are reached [see Fig. 4(c)]. The dependence of the resonance frequency as a function of the in-plane torque is shown in Fig. 4(d), where the positive slope is attributed to out-of-plane precessions, whereas the negative slope is attributed to in-plane precessions.

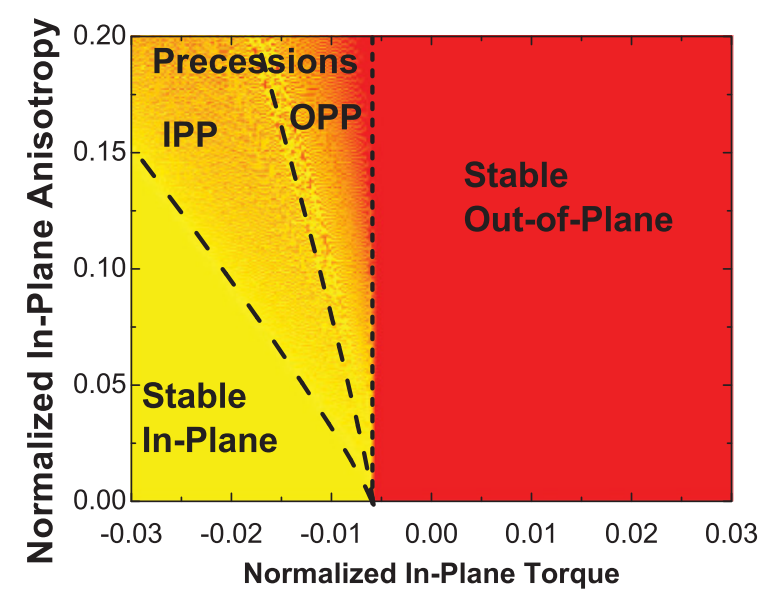

FIG. 3. (Color online) Stability diagram of the magnetization in the presence of perpendicular and in-plane anisotropy as well as in-plane torque. The parameters are magnetic damping $\eta=0.01$, perpendicular anisotropy $Q_{\perp}=0.75$, and saturation magnetization $M_{s}=1.6 \mathrm{~T}^{20}$. The dashed lines are guides for the eye.

The present current-driven spin torque can be readily compared with the conventional STT $^{1}$ and the SOI-ST. ${ }^{9,16}$ First, the present torque has its origin in the interfacial SOI, rather than in the inhomogeneous or discontinuous magnetic texture, so that no external polarizer is needed. Second, it possesses two components, $T_{\|}$and $T_{\perp}$, whereas the SOI-ST calculated in Refs. 9 and 16 produces only an effective magnetic field. Furthermore, similar to the conventional STT, the in-plane torque $T_{\|}$competes with the damping and can excite self-sustained magnetic precessions in the absence of external magnetic field, provided that in-plane and out-ofplane magnetization stable states can be achieved.

For the parameters exploited here, the spin torque can be as large as $100 \mathrm{eV} / \mu \mathrm{m}^{2}$ [see Fig. 2(b)], which is comparable to
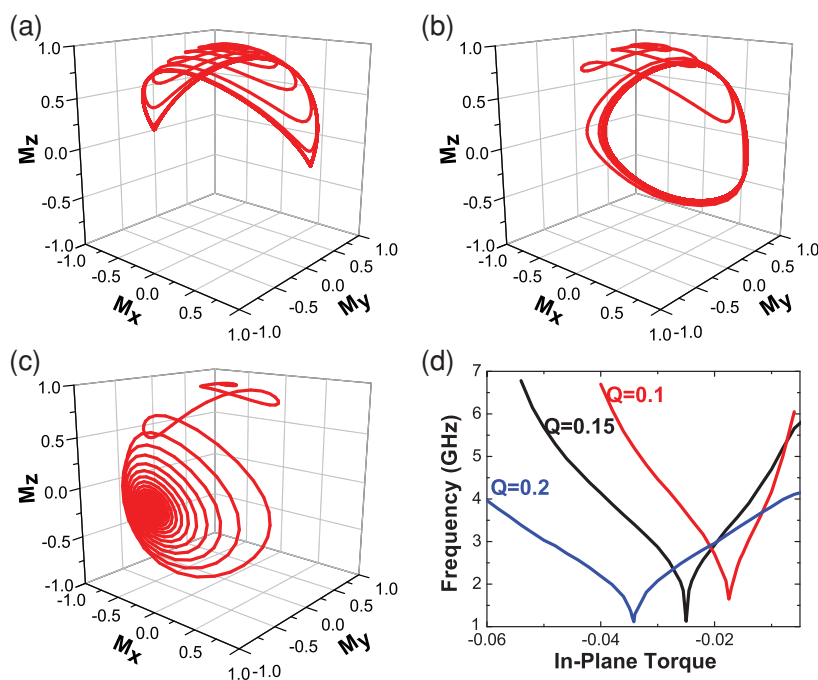

FIG. 4. (Color online) Magnetization trajectory for a magnetization initially perpendicular to the plane and submitted to an in-plane anisotropy $Q_{\|}=0.2$ and (a) $\tau_{\|}=-0.0125$, (b) $\tau_{\|}=-0.02$, and (c) $\tau_{\|}=-0.0375$. (d) Dependence of the oscillation frequency as a function of the in-plane torque. 
spin transfer torque in MTJs. ${ }^{17}$ From an experimental point of view, the key element needed to observe such spin torque is a large spin-orbit splitting at the interface between the ferromagnet and the barrier. As mentioned in the introduction, a number of results have been produced on SMTJs based on metals ${ }^{7}$ or semiconductors. ${ }^{5}$ A good rule of thumb to design such efficient interfaces is to maximize the TAMR, as shown in Ref. 7. An efficient procedure to detect such a torque in the case of small bias (or small Rashba parameter) is to investigate spin-torque-driven ferromagnetic resonance ${ }^{21}$ or spin-diode effects. $^{22}$

The author acknowledges fruitful discussions with S. Zhang, K.-J. Lee, and P. J. Kelly. This work was supported by the KAUST Academic Excellence Alliance funding program.
${ }^{1}$ J. C. Slonczewski, J. Magn. Magn. Mater. 159, L1 (1996);

L. Berger, Phys. Rev. B 54, 9353 (1996).

${ }^{2}$ M. Tsoi et al., Phys. Rev. Lett. 80, 4281 (1998); J. A. Katine et al., ibid. 84, 3149 (2000).

${ }^{3}$ J. A. Katine and E. E. Fullerton, J. Magn. Magn. Mater. 320, 1217 (2008).

${ }^{4}$ M. D. Stiles and J. Miltat, Top. Appl. Phys. 101, 225 (2006);

D. C. Ralph and M. D. Stiles, J. Magn. Magn. Mater. 320, 1190 (2008); J. Z. Sun and D. C. Ralph, ibid. 320, 1227 (2008).

${ }^{5}$ C. Gould et al., Phys. Rev. Lett. 93, 117203 (2004).

${ }^{6}$ J. Moser et al., Phys. Rev. Lett. 99, 056601 (2007); M. Wimmer et al., Phys. Rev. B 80, 121301(R) (2009).

${ }^{7}$ B. G. Park et al., Phys. Rev. Lett. 100, 087204 (2008).

${ }^{8}$ T. Uemura et al., Appl. Phys. Lett. 94, 182502 (2009).

${ }^{9}$ A. Manchon and S. Zhang, Phys. Rev. B 78, 212405 (2008); 79, 094422 (2009).

${ }^{10}$ I. M. Miron et al., Nat. Mater. 9, 230 (2010); U. H. Pi et al., Appl. Phys. Lett. 97, 162507 (2010).

${ }^{11}$ A. Chernyshov et al., Nat. Phys. 5, 656 (2009); M. Endo, F. Matsukura, and H. Ohno, Appl. Phys. Lett. 97, 222501 (2010).
${ }^{12}$ T. Maruyama et al., Nat. Nanotechnol. 4, 158 (2009); M. Endo et al., Appl. Phys. Lett. 96, 212503 (2010).

${ }^{13}$ Y. A. Bychkov and E. I. Rashba, J. Phys. C 17, 6039 (1984); G. Dresselhaus, Phys. Rev. 100, 580 (1955).

${ }^{14}$ J. Nitta et al., Phys. Rev. Lett. 78, 1335 (1997).

${ }^{15}$ O. Krupin et al., Phys. Rev. B 71, 201403(R) (2005); H. Cercellier et al., ibid. 73, 195413 (2006); C. R. Ast et al., Phys. Rev. Lett. 98, 186807 (2007).

${ }^{16}$ A. Matos-Abiague and R. L. Rodriguez-Suarez, Phys. Rev. B 80, 094424 (2009); I. Garate and A. H. MacDonald, ibid. 80, 134403 (2009); P. M. Haney and M. D. Stiles, Phys. Rev. Lett. 105, 126602 (2010).

${ }^{17}$ J. Xiao, G. E. W. Bauer, and A. Brataas, Phys. Rev. B 77, 224419 (2008).

${ }^{18}$ A. Matos-Abiague and J. Fabian, Phys. Rev. B 79, 155303 (2009); A. Matos-Abiague, M. Gmitra, and J. Fabian, ibid. 80, 045312 (2009).

${ }^{19}$ A. N. Chantis et al., Phys. Rev. Lett. 98, 046601 (2007).

${ }^{20}$ S. Ikeda et al., Nat. Mater. 9, 721 (2010).

${ }^{21}$ S. Petit et al., Phys. Rev. Lett. 98, 077203 (2007).

${ }^{22}$ H. Kubota et al., Nat. Phys. 4, 37 (2008); J. C. Sankey et al., ibid. 4, 67 (2008). 